\title{
Planejamento experimental do processo de secagem da amora-preta (Rubus sp.) para a produção de farinha enriquecida com compostos bioativos
}

\section{Experimental design of blackberry (Rubus sp.) drying process for production of flour enriched with bioactive compounds}

\author{
Fabiana Casarin'1, Caroline Eliza Mendes' ${ }^{1}$,Toni Jefferson Lopes², Neusa Fernandes de Moura2* \\ 'Universidade Comunitária da Região de Chapecó (UNOCHAPECO), Área de Ciências Exatas e Ambientais, Chapecó/SC - Brasil \\ 2Universidade Federal do Rio Grande (FURG), Escola de Química e Alimentos (EQA), Santo Antonio da Patrulha/RS - Brasil
}

\section{*Corresponding Author}

Neusa Fernandes de Moura, Universidade Federal do Rio Grande (FURG), Escola de Química e Alimentos (EQA), Rua Barão do Caí, 125, Cidade Alta, CEP: 955000-000, Santo Antonio da Patrulha/RS - Brasil, e-mail: nfmfurg@gmail.com

Cite as: Experimental design of blackberry (Rubus sp.) drying process for production of flour enriched with bioactive compounds. Braz. J. Food Technol., v. 19, e2016025, 2016.

\section{Resumo}

O estudo do processo de secagem foi realizado a partir da utilização de técnicas de planejamento experimental investigando os seguintes fatores: temperatura de secagem $\left(30\right.$ a $\left.80{ }^{\circ} \mathrm{C}\right)$, massa da amora $(6,0$ a $135 \mathrm{~g})$ e tempo ( 16 a $\left.24 \mathrm{~h}\right)$, tendo como variável de resposta a umidade e concentração dos compostos fenólicos. Também foram fixados os fatores: tipo de amostra (triturada) e estufa (com circulação de ar) para realização dos ensaios experimentais. Os resultados mostraram perdas pequenas de compostos bioativos no processo a $55^{\circ} \mathrm{C}$ por 16 horas com $80 \mathrm{~g}$ de amostra. De acordo com as análises químicas, a farinha encontra-se dentro dos padrões exigidos pela legislação brasileira para farinhas (máximo de 15\%), podendo ser classificada como produto de alto percentual de fibra $(24,33 \pm 2,88 \mathrm{~g} / 100 \mathrm{~g}$ de farinha). Foram determinadas na farinha através de métodos espectrofotométricos: Fenólicos Totais: 344,94 mg GAE/100 g; Flavonoides Totais 182,82 mg Ce/100 g; Antocianinas Totais 77,93 mg/100 g; Antocianinas Monoméricas 82,41 mg CYD-3-G/100 g; Carotenoides Totais $0,322 \mathrm{mg} / 100 \mathrm{~g}$; e a Atividade Antioxidante, utilizando o método DPPH, resultando em um EC en $_{\text {de }}$ $21,2 \mu \mathrm{g} / \mathrm{mL}$. Estes resultados credenciam a farinha como um produto rico em compostos bioativos, agregando valor aos alimentos e aos produtos obtidos a partir desta farinha.

Palavras-chave: Compostos fenólicos; Antocianinas; Análise estatística.

\section{Summary}

The blackberry drying process was studied using an experimental design with three factors, namely: sample weight (6 to $135 \mathrm{~g}$ ), drying temperature $\left(30\right.$ to $80^{\circ} \mathrm{C}$ ) and time (16 to $24 \mathrm{~h}$ ). All samples were grounded and dried accordingly, in a convection oven with air circulation. Moisture and phenolic compounds concentration were chosen as response variables. Results showed small losses of bioactive compounds for the $80 \mathrm{~g}$ sample dried at $55^{\circ} \mathrm{C}$ for 16 hours. Furthermore, the blackberry flour meets the Brazilian standards to moisture (maximum 15\%) and can be classified as a product of high fiber content $(24.33 \pm 2.88 \mathrm{~g} / 100 \mathrm{~g}$ of flour). Bioactive compounds in the flour were quantified using spectrophotometric analysis: total phenolic of $344.94 \mathrm{mg} \mathrm{GAE} / 100 \mathrm{~g}$; total flavonoids of $182.82 \mathrm{Ce} \mathrm{mg/100} \mathrm{g;} \mathrm{total} \mathrm{anthocyanins} \mathrm{of} 77.93 \mathrm{mg} / 100 \mathrm{~g}$; monomeric anthocyanins of $82.41 \mathrm{CYD}-3-\mathrm{G} / 100 \mathrm{~g}$; total carotenoids of $0.322 \mathrm{mg} / 100 \mathrm{~g}$ and activity anti-DPPH EC ${ }_{50} 21.2 \mu \mathrm{g} / \mathrm{mL}$. These results show that blackberry flour is rich in bioactive compounds, adding nutritional value to foods and products made with this flour.

Keywords: Phenolic compounds; Anthocyanins; Statistical analysis. 
Planejamento experimental do processo de secagem da amora-preta (Rubus sp.) para a produção de farinha enriquecida com compostos bioativos

Casarin, F. et al.

\section{Introdução}

A amora-preta é uma espécie típica de clima temperado e sua produção mundial ocupa cerca de 20 mil hectares, distribuídos em todos os continentes. Seu cultivo teve início no Brasil em meados da década de 1970 na cidade de Pelotas no Estado do Rio Grande do Sul (RASEIRA, 2004) e vem crescendo em diversos estados brasileiros, estimando-se que a área cultivada seja de aproximadamente 300 hectares, com produção de 780 toneladas/ano, sendo a cidade de Vacaria-RS o maior produtor brasileiro da fruta (ANTUNES, 2006; STRIK et al., 2007; TULLIO; AYUB, 2013).

A amoreira-preta apresenta frutas de sabor agridoce, com alto valor nutritivo e alta concentração de compostos bioativos, principalmente fenólicos, com destaque para os pigmentos antociânicos (ACOSTA-MONTOYA et al., 2010; FERRARI et al., 2012; ROMERO JUNIOR; VILLAREAL, 2015). Os compostos fenólicos são responsáveis pela pigmentação do vermelho ao azul nas frutas e atuam na saúde humana, no combate de doenças neurodegenerativas, na apoptose de células cancerígenas, como anti-inflamatório e antiproliferativo, possuindo também função antioxidante (JACQUES; ZAMBIAZI, 2011; FERRARI et al., 2012; REÁTEGUI et al., 2014).

A qualidade das frutas depende da matéria-prima e em grande parte das tecnologias empregadas na colheita e pós-colheita. Segundo Silva (2003), as técnicas utilizadas nesta fase não melhoram a qualidade da fruta, mas retardam o processo de senescência e decomposição da fruta, melhorando a sua conservação.

O processo de secagem é utilizado para minimizar as perdas pós-colheita, gerando produtos com maior valor nutritivo e estáveis (DOMINGUES, 2002; MARTINELLO et al., 2013). A otimização do processo de secagem de frutas é uma tecnologia clássica que vêm sendo constantemente estudada e aperfeiçoada, para obtenção de produtos com maior qualidade em menor tempo de processamento.

Assim sendo, o objetivo deste trabalho foi aperfeiçoar o processo de secagem da amora-preta em função da temperatura, tempo e massa de amostra, definindo a condição ótima para obtenção da farinha com alto valor de compostos fenólicos, que são os compostos bioativos majoritários na amora, sem perdas consideráveis de suas atividades funcionais.

\section{Material e métodos}

\subsection{Matéria-prima}

Foram adquiridas da empresa Fragoli Produtos Alimentícios, localizada na cidade de Pelotas - RS, no mês de novembro, dois lotes de $20 \mathrm{~kg}$ de amora-preta (Rubus sp.) (safra 2009 e 2010). As frutas inteiras e embaladas foram armazenadas em freezer a $-18{ }^{\circ} \mathrm{C}$.
As amoras da safra de 2009 foram utilizadas na determinação das condições operacionais do processo (umidade e compostos fenólicos) e, posteriormente, foram avaliadas a reprodutibilidade e a robustez do modelo empírico obtido nas condições otimizadas, na safra de 2010, em que se ampliou o estudo dos compostos presentes antes e após a secagem (umidade e compostos bioativos).

\subsection{Preparo da amostra}

Para realizar os experimentos, em escala de bancada, as amostras foram transferidas do freezer para um refrigerador $\left(5^{\circ} \mathrm{C}\right)$ durante 24 horas, para o processo de descongelamento. Depois deste período, as amoras foram lavadas em água corrente e imersas em solução de água clorada 15 ppm, por um período de 15 minutos. Posteriormente, as amostras foram inseridas em placas de petri e secas em estufas sem ou com circulação de ar forçada (velocidade do ar de $1,0 \mathrm{~m} / \mathrm{s}$ ). Na etapa em que se realizou o planejamento experimental, será descrita, com maiores detalhes, a etapa de secagem, sendo que foi estabelecida uma faixa de $5 \%$ até $15 \%$ para a umidade final do produto obtido, valor este estabelecido na legislação para farinhas (ANVISA, 1978).

\subsection{Planejamento experimental do processo de secagem}

No processo de secagem da amora-preta (amostra do ano de 2009 - concentração inicial de fenólicos de $581,24 \mathrm{mg} / 100 \mathrm{~g}$ em base seca), foi avaliada a influência de 3 variáveis independentes sobre a variável resposta, umidade e concentração de fenólicos. As variáveis independentes avaliadas foram: (1) temperatura de secagem, (2) massa da amostra e (3) tempo de processo.

A temperatura e o tempo são fatores determinantes, pois influenciam significativamente o processo de secagem e estes fatores favorecem a decomposição de compostos fenólicos (GARAU et al., 2007; OLIVEIRA et al., 2009). Assim, foi definida através de ensaios experimentais preliminares a temperatura mínima de $30^{\circ} \mathrm{C}$ até a máxima de $80^{\circ} \mathrm{C}$; e tempos de 16 a $24 \mathrm{~h}$ para obtenção de teores de umidade entre $5 \%$ e $15 \%$ e maior concentração de fenólicos no produto final obtido no processo. O terceiro fator estudado foi a quantidade de amostra, 6 a $135 \mathrm{~g}$; como critério de escolha foi considerada, principalmente, que a transferência de um líquido ou vapor de um sólido é influenciada pela quantidade de massa utilizada durante o processo de secagem.

Outros dois fatores do processo foram fixados: tipo de estufa com circulação de ar e a forma da amostra triturada, fator este importante no processo de secagem, pois quanto maior a superfície de contato maior a perda de água durante a secagem. 
Planejamento experimental do processo de secagem da amora-preta (Rubus sp.) para a produção de farinha enriquecida com compostos bioativos

Casarin, F. et al.

Posterior à seleção das variáveis, foi determinado o tipo de planejamento com configuração estrela e os níveis do estudo, cinco níveis codificados foram utilizados $(-\alpha,-1,0,+1 e+\alpha)$. Os pontos axiais $( \pm \alpha)$ foram utilizados para a ampliação do modelo linear, permitindo testar modelos empíricos quadráticos. Os valores codificados dos níveis $+\alpha$ e $-\alpha$ foram obtidos pela Equação 1, onde o valor de $\alpha$ determinado foi de 1,68.

$$
\alpha=\sqrt[4]{2^{n}}
$$

onde: n é o número de fatores (3).

As variáveis e seus respectivos níveis de variação são mostrados na Tabela 1. Posteriormente à realização do planejamento em estrela, aplicou-se a Metodologia das Superfícies de Resposta (MSR) com o objetivo de obter as condições ótimas de operação através do software Statistica 6.0.

\subsection{Avaliação dos compostos bioativos e caracterização química da amora-preta}

Os ensaios experimentais foram realizados para a avaliação das características químicas (base úmida para amora in natura e base seca/úmida para a farinha) e dos compostos bioativos (base seca) das amostras de amora in natura e do produto obtido nas condições otimizadas de operação de secagem (amostra de amora-preta do ano de 2010). Os testes foram feitos em triplicata e analisados estatisticamente através do teste de médias de Tukey $(p \leq 0,05)$.

\subsection{Determinação dos compostos fenólicos}

Os compostos fenólicos foram extraídos segundo o método de Kim et al. (2003). Foram utilizados $10 \mathrm{~g}$ de amora-preta in natura ou na forma de farinha para extração com uma solução de $45 \%$ de metanol, $45 \%$ de etanol e $10 \%$ de acetona, em agitação por 60 minutos. A amora com a solução extratora foi filtrada em funil de Büchner e lavada com $50 \mathrm{~mL}$ de metanol. A extração foi repetida exaustivamente e o filtrado transferido a um evaporador rotatório a $40{ }^{\circ} \mathrm{C}$ (Quimis, modelo: Q344B). O extrato concentrado foi dissolvido em $50 \mathrm{~mL}$ de metanol e o volume completado com água destilada até $100 \mathrm{~mL}$. A solução foi então centrifugada (HETTICH, modelo: EBA 270) por 20 minutos e armazenada em freezer $\left(-18^{\circ} \mathrm{C}\right)$ até a realização da análise.
A determinação dos teores de compostos fenólicos totais foi realizada pelo método de Folin-Denis, de acordo com o método 9110 da AOAC (HOROWITZ, 2005). A curva de calibração utilizada foi de ácido gálico e as leituras da absorbância realizadas em espectrofotômetro de UV visível (Marca SCINCOSUV, Modelo 2120) a $760 \mathrm{~nm}$. Os resultados foram expressos em mg de ácido gálico/100 g (base seca).

\subsection{Análise de flavonoides totais}

Para a análise de flavonoides totais presentes na amora-preta seca e in natura, foi seguida a metodologia descrita por Zhishen et al. (1999). Para a preparação da curva de calibração foi utilizado catecol como padrão e a leitura da absorbância foi realizada a $510 \mathrm{~nm}$ em espectrofotômetro de UV visível. Os resultados foram expressos em mg de catecol/100 g (base seca).

\subsection{Antocianinas totais}

Foram pesados $2 \mathrm{~g}$ de amora-preta em um béquer de $100 \mathrm{~mL}$ e adicionados $10 \mathrm{~mL}$ da solução extratora [etanol 95\%/HCl 1,5 mol/L (85:15)]. A mistura foi submetida à agitação magnética por 5 minutos, armazenada sob ausência de luz a $4{ }^{\circ} \mathrm{C}$ por 16 horas. A seguir, foi filtrada em funil e o resíduo foi lavado com a solução extratora e transferido para um balão volumétrico de $100 \mathrm{~mL}$. Depois de ajustar o volume com o solvente extrator, a solução de antocianinas obtida foi deixada em repouso por 60 minutos e então foi realizada a leitura em espectrofotômetro a $528 \mathrm{~nm}$. O cálculo realizado do teor de antocianinas totais foi expresso em equivalente da antocianina principal, cianidina-3-glucosídeo, segundo metodologia de Fuleki e Francis (1968).

\subsection{Antocianinas monoméricas}

Para análise de antocianinas monoméricas, utilizou-se o método descrito por Lee et al. (2005). Foram pesados 2 g de amora-preta e extraídos com solução metanólica, acidificada com $\mathrm{HCl}$ 1\%, e submetido a agitação por 1 hora. A seguir, a solução foi filtrada e concentrada em evaporador rotatório, obtendo-se, desta forma, o extrato bruto. O extrato foi diluído em água destilada até $25 \mathrm{~mL}$. Foram preparadas duas soluções com a mesma quantidade de extrato diluído, uma em tampão pH 1,0 (cloreto de potássio 0,025 M) e outra em tampão pH 4,5 (acetato de sódio 0,4 M), as quais

Tabela 1. Fatores e níveis estudados durante a triagem no planejamento experimental.

\begin{tabular}{lccccc}
\multicolumn{1}{r}{ Fatores } & \multicolumn{4}{c}{ Níveis de variação dos fatores } \\
\cline { 2 - 6 } & $\mathbf{- 1 , 6 8}$ & $\mathbf{- 1}$ & $\mathbf{0}$ & $\mathbf{+ 1}$ & $\mathbf{- 1 , 6 8}$ \\
* Temperatura $\left({ }^{\circ} \mathrm{C}\right)$ & 30 & 40 & 55 & 70 & 80 \\
${ }^{*}$ Massa de Amora $(\mathrm{g})$ & 6,0 & 30 & 65 & 100 & 135 \\
* Tempo $(\mathrm{h})$ & 16 & 18 & 20 & 22 & 24 \\
\hline
\end{tabular}

*Fatores fixados com base em resultados de testes preliminares. 
Planejamento experimental do processo de secagem da amora-preta (Rubus sp.) para a produção de farinha enriquecida com compostos bioativos

Casarin, F. et al.

ficaram em repouso por 30 minutos na ausência de luz. Em seguida, foram realizadas as leituras da absorbância em espectrofotômetro UV visível (marca SCINCOSUV, modelo 2120). A quantificação de antocianinas monoméricas foi obtida a partir do cálculo da diferença entre os tampões em comprimentos de onda 520 e $720 \mathrm{~nm}$.

\subsection{Carotenoides totais}

Foram utilizados $5 \mathrm{~g}$ de amora-preta e extraídos com 10 mL de solução acetona:hexano (4:6 v/v) sob agitação. $\mathrm{O}$ sobrenadante foi centrifugado por $5 \mathrm{~min}$. A leitura das absorbâncias foi realizada em espectrofotômetro a 450nm. A determinação da concentração dos carotenoides totais foi realizada de acordo com Mercadante et al. (1997).

\subsection{Determinação do radical DPPH (2,2-difenil-1- picrilhidrazila)}

O método utilizado para a determinação da atividade antioxidante via radical DPPH (2,2-difenil-1-picrilhidrazila) foi desenvolvido por Brand-Williams et al. (1995), com modificações por Mensor et al. (2001). O resultado foi expresso em percentagem de atividade antioxidante (AA\%). Os dados do método DPPH foram também interpretados pelo $\mathrm{CE}_{50}$, ou seja, a concentração da amostra em que $50 \%$ da atividade antioxidante (antirradical) foi observada.

\subsection{Caracterização química da farinha de amora}

Foram determinados os teores de umidade, cinzas, proteínas, fibras brutas, carboidratos e lipídeos de acordo com as respectivas metodologias da AOAC (HOROWITZ, 2005).

\section{Resultados e discussão}

A primeira etapa do estudo foi a realização do planejamento estrela, com três fatores e com triplicata no ponto central (Tabela 2), com o intuito de obter uma região ótima de processamento do alimento.

\subsection{Estudo do ponto ótimo de secagem para a variável resposta umidade da farinha de amora-preta}

Primeiramente, avaliaram-se os valores de $\mathrm{p}$ (probabilidade) dos efeitos estimados de cada variável estudada (temperatura, tempo e massa), para o termo linear e quadrático do tempo, obtiveram-se os valores de 0,1306 e 0,0921, respectivamente. Portanto, valores superiores ao do nível de confiança p escolhido de 0,05, consequentemente, um fator que não apresenta significância em relação aos fatores temperatura e massa (valores de p inferiores a 0,05). Visto que o tempo é um fator que não pode ser excluído do sistema, fixou-se o valor do menor nível (16 horas) para obtenção das condições ótimas do processo em estudo. Posteriormente, obteve-se o modelo empírico codificado para representar a umidade da amora-preta ao final do tempo de processo, Equação 2.

$y=6,15-2,84 T+1,24 T^{2}+0,97 M+0,85 M^{2}$

onde: y é o percentual de umidade ajustado, T é a temperatura e M é a massa de amoras.

$O$ valor do coeficiente de determinação $\mathrm{R}^{2}$ encontrado foi $84,44 \%$, indicando que um modelo quadrático representa bem a relação entre os efeitos e a resposta. Também foi

Tabela 2. Planejamento estrela para temperatura, massa e tempo.

\begin{tabular}{|c|c|c|c|c|c|c|c|c|}
\hline \multirow[b]{2}{*}{ Ensaio } & \multicolumn{3}{|c|}{ Tabela codificada } & \multicolumn{3}{|c|}{ Tabela decodificada } & \multicolumn{2}{|c|}{ Resposta } \\
\hline & $\begin{array}{c}\text { Temperatura } \\
\left({ }^{\circ} \mathrm{C}\right)\end{array}$ & $\begin{array}{c}\text { Massa } \\
\text { (g) }\end{array}$ & $\begin{array}{c}\text { Tempo } \\
\text { (h) }\end{array}$ & $\begin{array}{c}\text { Temperatura } \\
\left({ }^{\circ} \mathbf{C}\right)\end{array}$ & $\begin{array}{c}\text { Massa } \\
\text { (g) }\end{array}$ & $\begin{array}{c}\text { Tempo } \\
\text { (h) }\end{array}$ & $\begin{array}{c}\text { Umidade } \\
\%\end{array}$ & $\begin{array}{l}\text { Fenólicos } \\
\mathrm{mg} / 100 \mathrm{~g}\end{array}$ \\
\hline 1 & -1 & -1 & -1 & 40 & 30 & 18 & 11,434 & 406,5 \\
\hline 2 & 1 & -1 & -1 & 70 & 30 & 18 & 5,840 & 278,7 \\
\hline 3 & -1 & 1 & -1 & 40 & 100 & 18 & 13,587 & 575,4 \\
\hline 4 & 1 & 1 & -1 & 70 & 100 & 18 & 5,951 & 238,8 \\
\hline 5 & -1 & -1 & 1 & 40 & 30 & 22 & 10,424 & 461,48 \\
\hline 6 & 1 & -1 & 1 & 70 & 30 & 22 & 6,588 & 171,6 \\
\hline 7 & -1 & 1 & 1 & 40 & 100 & 22 & 12,045 & 457,2 \\
\hline 8 & 1 & 1 & 1 & 70 & 100 & 22 & 6,434 & 401,4 \\
\hline 9 & $-1,68$ & 0 & 0 & 30 & 65 & 20 & 13,181 & 477 \\
\hline 10 & 1,68 & 0 & 0 & 80 & 65 & 20 & 3,677 & 87 \\
\hline 11 & 0 & $-1,68$ & 0 & 55 & 6 & 20 & 4,623 & 191,1 \\
\hline 12 & 0 & 1,68 & 0 & 55 & 135 & 20 & 11,534 & 434,7 \\
\hline 13 & 0 & 0 & $-1,68$ & 55 & 65 & 16 & 8,157 & 494,7 \\
\hline 14 & 0 & 0 & 1,68 & 55 & 65 & 24 & 4,819 & 371,7 \\
\hline 15 & 0 & 0 & 0 & 55 & 65 & 20 & 5,595 & 431,1 \\
\hline 16 & 0 & 0 & 0 & 55 & 65 & 20 & 4,463 & 435,6 \\
\hline 17 & 0 & 0 & 0 & 55 & 65 & 20 & 5,891 & 420,3 \\
\hline
\end{tabular}


Planejamento experimental do processo de secagem da amora-preta (Rubus sp.) para a produção de farinha enriquecida com compostos bioativos

Casarin, F. et al.

observado pela análise de variância (Tabela 3), que os valores da distribuição $F_{\text {calculados }}$ para os resíduos em relação à regressão é 5,0 vezes superior ao valor da distribuição $F_{\text {tabelado }}$ em um nível de confiança de 95\% do processo. Deste modo, entende-se que a regressão é significativa, podendo ser usada para fins de predição (BOX et al., 2005). Do mesmo modo, os valores dos resíduos em relação à regressão não são significativos. Portanto, o modelo ajustado (Equação 2) mostra-se adequado para fins preditivos.

Na Figura 1 são apresentadas as curvas de nível do modelo proposto (Equação 2) para as respostas geradas a partir dos dados experimentais, obtidos para o processo de secagem da amora-preta. Pode-se observar que a região delimitada pelo gráfico apresenta os teores de umidade requeridos para as farinhas, entre $5 \%$ e $15 \%$.

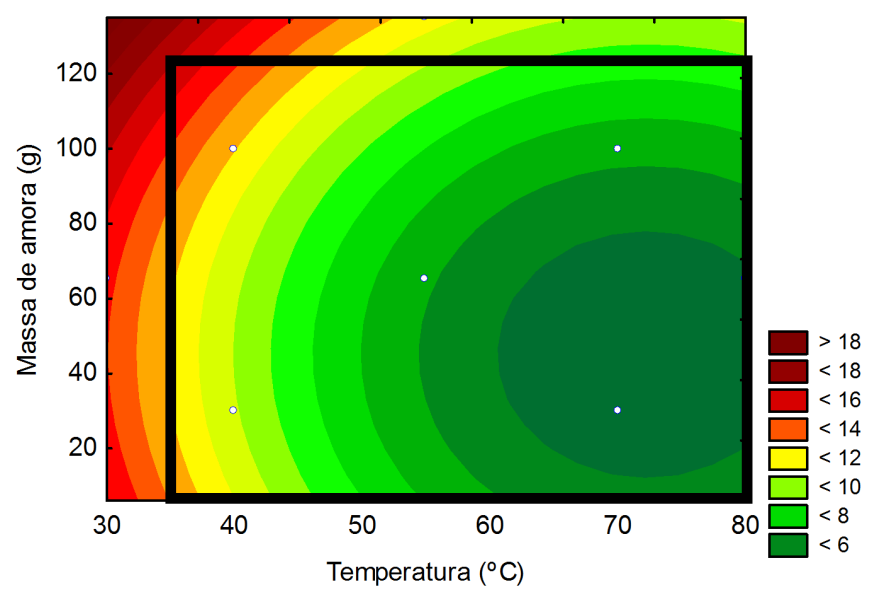

Figura 1. Gráfico de curvas de contorno para os fatores massa (g) e temperatura $\left({ }^{\circ} \mathrm{C}\right)$ para umidade.

\subsection{Estudo do ponto ótimo de secagem em função da concentração dos compostos fenólicos na farinha de amora-preta}

O modelo empírico codificado, proposto para representar a concentração de compostos fenólicos na amora-preta, está representado pela Equação 3.

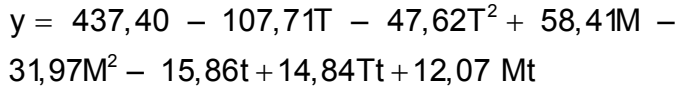

onde: y é a concentração de compostos fenólicos, T é a temperatura, t é o tempo e M é a massa de amoras.

$\mathrm{O}$ coeficiente de determinação $\mathrm{R}^{2}$ do processo foi de $86,61 \%$, indicando que um modelo quadrático representa bem a relação entre os efeitos e resposta. Também foi observado, pela análise de variância (Tabela 4), que o modelo empírico para a concentração dos compostos fenólicos da farinha de amora-preta não se mostra perfeitamente ajustado aos dados experimentais, conforme pode-se observar no comparativo entre os valores de $\mathrm{F}_{\text {calculado }}$ e $\mathrm{Ft}_{\text {abelado }}$. Porém, o modelo pode ser utilizado para descrever a tendência dos resultados obtidos, com limitações para fins preditivos.

A Figura 2 apresenta as curvas de contorno do modelo proposto para a resposta gerada, a partir dos dados experimentais da concentração de compostos fenólicos.

Na avaliação conjunta das respostas das Figuras 1 e 2, verifica-se que, para a faixa estudada, com máximo de $15 \%$ de umidade em farinhas e maior concentração de compostos fenólicos, as condições adequadas foram: $55^{\circ} \mathrm{C}$ por 16 horas, em estufa com circulação de ar, para uma massa de $80 \mathrm{~g}$ da fruta triturada. Em estudo realizado por Ferrari et al. (2013), foram obtidos valores

Tabela 3. Análise de variância para a umidade da amora-preta (ANOVA)

\begin{tabular}{lccccc} 
Fonte de variação & Soma Quadrática & $\begin{array}{c}\text { Graus de } \\
\text { Liberdade }\end{array}$ & $\begin{array}{c}\text { Médias } \\
\text { Quadráticas }\end{array}$ & $\begin{array}{c}\text { F } \\
\text { calculado }\end{array}$ & $\begin{array}{c}\text { F } \\
\text { tabelado }\end{array}$ \\
\hline Regressão & 153,5575 & 4 & 38,38937039 & 16,39 & 3,26 \\
Resíduos & 28,1013 & 12 & 2,341772319 & & 19,4 \\
Falta de ajuste & 26,9652 & 10 & 2,696519316 & 4,75 & \\
Erro Puro & 1,1361 & 2 & 0,568037333 & & \\
Total & 209,7600 & & & & \\
\hline
\end{tabular}

Tabela 4. Análise de variância para a concentração de compostos fenólicos na farinha de amora-preta (ANOVA).

\begin{tabular}{lccccc} 
Fonte de variação & Soma Quadrática & $\begin{array}{c}\text { Graus de } \\
\text { Liberdade }\end{array}$ & $\begin{array}{c}\text { Médias } \\
\text { Quadráticas }\end{array}$ & $\begin{array}{c}\text { F } \\
\text { Calculado }\end{array}$ & $\begin{array}{c}\text { Tabelado } \\
\text { Regressão }\end{array}$ \\
Resíduos & 257909,8 & 7,0 & 36844,26 & 8,87 & 3,29 \\
Falta de ajuste & 37372,9 & 9,0 & 4152,543 & & 19,35 \\
Erro Puro & 37249,2 & 7,0 & 5321,318 & 86,06 & \\
Total & 123,7 & 2,0 & 61,83 & & \\
\hline
\end{tabular}


Planejamento experimental do processo de secagem da amora-preta (Rubus sp.) para a produção de farinha enriquecida com compostos bioativos

Casarin, F. et al.

menores de umidade, de 1,74\% a 3,32\% com spray-dried, porém ocorreu a necessidade de utilização de agentes carreadores. Segundo Ferrari et al. (2012), o suco de frutas, devido ao seu alto conteúdo de açúcares pode acarretar a obtenção de produtos com alta pegajosidade e higroscopicidade, diminuindo o rendimento do processo; os agentes carreadores auxiliam no transporte e no aumento do rendimento.

\subsection{Compostos bioativos na farinha de amora-preta}

A avaliação das características físicas e químicas do produto obtido, bem como a avaliação dos compostos bioativos foram realizadas nas condições anteriormente definidas para o processamento de secagem (Tabela 5).

A Tabela 5 apresenta os resultados encontrados para os compostos bioativos na amora antes e após o processo de secagem (dentro da melhor condição obtida para secagem). Também pode-se observar que os valores de fenólicos totais são semelhantes aos reportados em outros estudos, a concentração de fenólicos variou de 261,95 a 929,62 mg AG/100 g (EMBRAPA, 2008).

O mesmo ocorreu para a concentração de flavonoides totais na amora-preta, que está dentro da

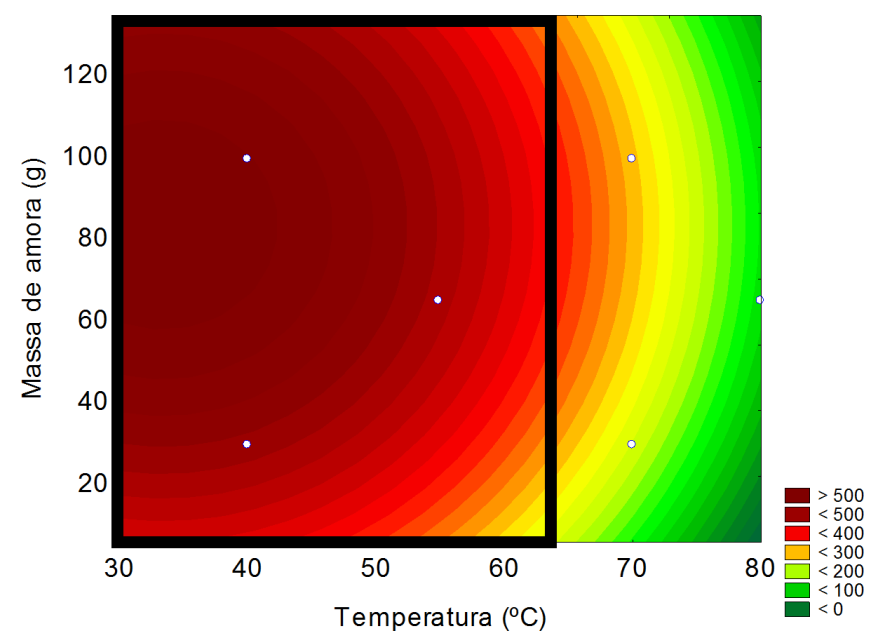

Figura 2. Gráfico de curvas de contorno para os fatores massa (g) e temperatura $\left({ }^{\circ} \mathrm{C}\right)$ para concentração dos compostos fenólicos. faixa encontrada na literatura de 123,3 a 213,3 mg/100 g (HASSIMOTTO et al., 2008). Com relação ao teor de antocianinas totais, as concentrações foram inferiores às observadas por Hassimotto et al. (2008), que foram superiores a $91 \mathrm{mg} / 100 \mathrm{~g}$. Porém, para as antocianinas monoméricas encontradas neste estudo, a concentração está dentro da ampla faixa encontrada em estudos anteriores (WANG et al., 2008). As antocianinas encontram-se comumente na forma de uma mistura de diferentes estruturas químicas em equilíbrio: cátion flavilium (vermelho), base anidra quinoidal (azul), pseudobase carbitol (incolor), e chalcona (incolor ou levemente amarela), sendo que a temperatura pode ocasionar maior ou menor grau de degradação das antocianinas, devido à estrutura da molécula do corante na amora, presença ou não de fatores como acilação da molécula de antocianinas ou copigmentos (OREN-SHAMIR, 2009)

A atividade antioxidante está relacionada à concentração de compostos bioativos presentes, principalmente com o conteúdo total de antocianinas (HASSIMOTTO et al., 2008). Como pode ser observado na Tabela 5, houve diferença significativa no teor de fitocompostos e atividade antioxidante, exceto para os carotenoides totais.

\subsection{Características químicas da farinha de amora-preta}

Os resultados da caracterização química da amora-preta seca (base seca e úmida) e in natura (base úmida) estão representados na Tabela 6 . Observa-se que o teor de umidade obtido para a farinha encontra-se dentro do padrão ANVISA (1978). Em farinhas com umidade acima de 14\% há a possibilidade do crescimento de microrganismos, como fungos filamentosos (bolores) e leveduras, além da diminuição da estabilidade e da vida útil da farinha (SGARBIERI, 1986).

O alto teor de fibras encontrado no fruto de amora, na faixa de $4 \%$ de fibras totais (base úmida), torna a farinha um produto indicado para ser adicionado na fabricação de produtos destinados a consumidores que buscam alimentos saudáveis, pois propiciam uma série de efeitos benéficos para a saúde, como a regularização do funcionamento intestinal e redução do colesterol plasmático

Tabela 5. Teores de compostos bioativos encontrados em amora-preta nas condições ótimas de secagem.

\begin{tabular}{lcc} 
& Amora-preta in natura & Farinha de amora-preta \\
Compostos Fenólicos Totais $(\mathrm{mg} \mathrm{GAE} / 100 \mathrm{~g})$ & $357,86 \pm 0,08^{\mathrm{A}}$ & $344,94 \pm 0,01^{\mathrm{B}}$ \\
Flavonoides Totais $(\mathrm{mg} \mathrm{Ce} / 100 \mathrm{~g})$ & $201 \pm 0,9^{\mathrm{A}}$ & $182,82 \pm 1,56^{\mathrm{B}}$ \\
Antocianinas Totais $(\mathrm{mg} / 100 \mathrm{~g})$ & $89,019 \pm 1,4^{\mathrm{A}}$ & $77,93 \pm 3^{\mathrm{B}}$ \\
Antocianinas Monoméricas $(\mathrm{mg} \mathrm{CYD}-3-\mathrm{G} / 100 \mathrm{~g})$ & $94,2 \pm 0,35^{\mathrm{A}}$ & $82,41 \pm 1,2^{\mathrm{B}}$ \\
Carotenoides Totais $(\mathrm{mg} / 100 \mathrm{~g})$ & $0,335 \pm 0,2^{\mathrm{A}}$ & $0,322 \pm 0,15^{\mathrm{A}}$ \\
Atividade Antirradical DPPH $\left(\mu \mathrm{g} / \mathrm{mL} \mathrm{DPPH} \mathrm{CE}_{50}\right.$ ) & $25,8 \pm 0,8^{\mathrm{A}}$ & $21,2^{\mathrm{A}} \pm 1,06^{\mathrm{B}}$ \\
\hline
\end{tabular}

\pm Média e desvio padrão de análise em triplicata; GAE - Valores expressos em equivalente de ácido gálico; Ce - Valores expressos em equivalente de catecol; GYD-3-G - Cianidina-3-glucosídeo $\mu \mathrm{g}$ amostra/g DPPH. Letras iguais na mesma linha não apresentam diferença significativa entre as médias pelo teste de Tukey $(\mathrm{p} \leq 0,05)$ 
Planejamento experimental do processo de secagem da amora-preta (Rubus sp.) para a produção de farinha enriquecida com compostos bioativos

Casarin, F. et al.

Tabela 6. Características da amora-preta in natura e da farinha de amora-preta.

\begin{tabular}{|c|c|c|c|}
\hline & Amora-preta (Base Úmida) & Farinha de amora-preta & Literatura \\
\hline Umidade & $80,56 \pm 0,4^{A}$ & $5,37 \pm 0,12$ & $\begin{array}{l}\text { Padrão ANVISA (1978) que exige o } \\
\text { máximo de umidade de } 15 \mathrm{~g} / 100 \mathrm{~g} \\
\text { de farinha }\end{array}$ \\
\hline Proteínas & $0,9 \pm 0,1^{A}$ & $\begin{array}{l}3,084 \pm 0,02 \text { B.S. } \\
0,60 \pm 0,0038{ }^{\text {в }} \text { B.U. }\end{array}$ & $\begin{array}{l}0,8 \% \text { na fruta } \\
\text { Antunes (2002) }\end{array}$ \\
\hline Lipídios & $0,087 \pm 0,002^{A}$ & $\begin{array}{l}0,71 \pm 0,23 \text { B.S. } \\
0,13 \pm 0,044^{A} \text { B.U. }\end{array}$ & $\begin{array}{l}0,15 \% \text { na fruta } \\
\text { Antunes (2002) }\end{array}$ \\
\hline Carboidratos & $13,94 \pm 0,54 \mathrm{~A}$ & $\begin{array}{l}66,51 \pm 2,25 \text { B.S. } \\
12,92 \pm 0,43^{A} \text { B.U. }\end{array}$ & $\begin{array}{l}85 \% \text { de água e } 10 \% \text { de } \\
\text { carboidratos na fruta (ANTUNES, } \\
\text { 2006). }\end{array}$ \\
\hline Fibras Totais & $4,08 \pm 0,11^{\mathrm{A}}$ & $\begin{array}{l}24,33 \pm 2,88 \text { B.S. } \\
4,73 \pm 0,55^{A} \text { B.U. }\end{array}$ & $\begin{array}{l}\text { Farinha de casca de batata, } \\
\text { Fernandes et al. (2008) } \\
1,46 \% \text { de fibra bruta }\end{array}$ \\
\hline Cinzas & $0,58 \pm 0,11^{\mathrm{A}}$ & $\begin{array}{l}3,54 \pm 0,08 \text { B.S. } \\
0,68 \pm 0,015 \text { A B.U. }\end{array}$ & --- \\
\hline
\end{tabular}

Média e desvio padrão de análise em triplicata. Letras diferentes na mesma linha apresentam diferença significativa das médias pelo teste de Tukey $(p \leq 0,05)$. B.S. representa a Base Seca e B.U. representa a Base úmida dos valores apresentados.

(CAVALCANTI, 1989). Os demais componentes da amora (Tabela 6) não apresentaram diferença significativa entre seu conteúdo antes e após o processo de secagem.

\section{Conclusão}

A técnica de planejamento experimental permitiu determinar as melhores condições de secagem da amora-preta em escala de bancada, quais foram: $55^{\circ} \mathrm{C}$ por 16 horas em estufa com circulação de ar, para uma massa de $80 \mathrm{~g}$ da amora-preta triturada. No entanto, para fins industriais, será preciso fazer uma avaliação ou uma extrapolação, para um processo produtivo, no qual as quantidades de material são muito maiores.

A farinha de amora-preta obtida pode apresentar características funcionais, devido ao seu alto teor de compostos fenólicos além de possuir atividade antioxidante, com potencial para obtenção de produtos alimentícios ricos em compostos bioativos.

O estudo realizado permitiu avaliar que a secagem é uma forma eficiente de conservação da amora-preta, permitindo a obtenção de farinha rica em compostos bioativos. Também servirá de base para o desenvolvimento de um processo tecnológico de produção, em futuros estudos em escala piloto ou industrial, para obtenção de um novo produto alimentício no mercado em época de entressafra, quando a fruta não está disponível no mercado.

\section{Referências}

ACOSTA-MONTOYA, O.; VAILLANT, F.; COZZANO, S.; MERTZ, C.; PEREZ, A. M.; CASTRO, M. V. Phenolic content and antioxidant capacity of tropical highland blackberry (Rubus adenotrichus
Schltdl.) during three edible maturity stages. Food Chemistry, London, v. 119, n. 4, p. 1497-1501, 2010. http://dx.doi.org/10.1016/j. foodchem.2009.09.032.

AGÊNCIA NACIONAL DE VIGILÂNCIA SANITÁRIA - ANVISA. Resolução CNNPA n. 12 de 1978. Diário Oficial [da] República Federativa do Brasil, Brasília, DF, 24 jul. 1978. Disponível em: <http://www.anvisa.gov.br>. Acesso em: 03 out. 2014.

ANTUNES, L. E. Amora-preta: nova opção de cultivo no Brasil. Ciência Rural, Santa Maria, v. 32, n. 1, p. 151-158, 2002. http:// dx.doi.org/10.1590/S0103-84782002000100026.

ANTUNES, L. E. Amora-preta (Rubus spp). Revista Brasileira de Fruticultura, Jaboticabal, v. 28, n. 3, p. 339-558, 2006.

BOX, G. E. P.; HUNTER, J. S.; HUNTER, W. G. Statistics for experimenters: design, innovation and discovery. 2. ed. New Jersey: Wiley-Interscience, 2005. 633 p.

BRAND-WILLIAMS, W.; CUVELIER, M. E.; BERSET, C. Use of free radical method evaluate antioxidant activity. LWT - Food Science and Technology, London, v. 28, n. 1, p. 25-30, 1995. http://dx.doi.org/10.1016/S0023-6438(95)80008-5.

CAVALCANTI, M. L. F. Fibras alimentares. Revista Nutrição, Campinas, v. 2, n. 1, p. 88-97, 1989.

DOMINGUES, A. Caracterização das propriedades físicas do suco de abacaxi (Ananás comosus) em pó desidratado por spray dryer otimizado através de análise de suporte de superfície de resposta. In: CONGRESSO BRASILEIRO DE CIÊNCIA E TECNOLOGIA DE ALIMENTOS, 2002, Porto Alegre. Anais.. Porto Alegre: CBCTA, 2002. p. 1717-1720.

EMPRESA BRASILEIRA DE PESQUISA AGROPECUÁRIA EMBRAPA. Embrapa Clima Temperado Sistemas de Produção. 
Planejamento experimental do processo de secagem da amora-preta (Rubus sp.) para a produção de farinha enriquecida com compostos bioativos

Casarin, F. et al.

Brasília: EMBRAPA, 2008. Disponível em: <http://www.embrapa. br>. Acesso em: 20 out. 2008.

FERNANDES, A.; PEREIRA, J.; GERMANI, R.; OIANO-NETO, J. Efeito da substituição parcial da farinha de trigo por farinha de casca de batata (Solanum Tuberosum Lineu). Ciência e Tecnologia de Alimentos, Campinas, v. 28, p. 56-65, 2008. http://dx.doi.org/10.1590/S0101-20612008000500010.

FERRARI, C. C.; GERMER, S. P. M.; ALVIM, I. D.; AGUIRRE, J. M. Storage stability of spray-dried blackberry powder produced with maltodextrin or gum Arabic. Drying Technology, Philadelphia, v. 31 , n. 4, p. 470-478, 2013. http://dx.doi.org/10.1080/073739 37.2012 .742103

FERRARI, C. C.; GERMER, S. P. M.; ALVIM, I. D.; VISSOTTO, F. Z.; AGUIRRE, J. M. Influence of carrier agents on the physicochemical properties of blackberry powder produced by spray drying. International Journal of Food Science \& Technology, Malden, v. 47, n. 6, p. 1237-1245, 2012. http://dx.doi.org/10.1111/j.13652621.2012.02964.x.

FULEKI, T.; FRANCIS, F. J. Quantitative methods for anthocyanins: determination of total anthocyanin and degradation index for cranberries juices. Journal of Food Science, Malden, v. 33, n. 1, p. 78-83, 1968. http://dx.doi.org/10.1111/j.1365-2621.1968. tb00888.x.

GARAU, M. C.; SIMAL, S.; ROSSELLÓ, C.; FEMENIA, A. Effect o fair-drying temperature on physico-chemical properties of dietary fibre and antioxidante capacity of Orange (Citrus auratium v. Canoneta) by-products. Food Chemistry, London, v. 104, n. 3, p. 1014-1024, 2007. http://dx.doi.org/10.1016/j. foodchem.2007.01.009.

HASSIMOTTO, N. M. A.; MOTA, R. V.; CORDENUNSI, B. R.; LAJOLO, F. M. Physico-chemical characterization and bioactive compounds of blackberry fruits (Rubus sp.) grown in Brazil. Ciência e Tecnologia de Alimentos, Campinas, v. 28, n. 3, p. 702-708, 2008. http://dx.doi.org/10.1590/S0101-20612008000300029.

HOROWITZ, W. (Ed.). Official methods of analysis of the Association Analytical Chemists. 18. ed. Gaithersburg: AOAC, 2005.

JACQUES, A. C.; ZAMBIAZI, R. C. Fitoquímicos em amora-preta (Rubus spp). Semina: Ciências Agrárias, Londrina, v. 32, n. 1, p. 245-260, 2011.

KIM, D. O.; JEONG, S. W.; LEE, C. Y. Antioxidant capacity of phenolic phytochemicals from various cultivars of plums. Food Chemistry, London, v. 81, n. 3, p. 321-326, 2003. http://dx.doi. org/10.1016/S0308-8146(02)00423-5.

LEE, S. J.; UMANO, K.; SHIBAMOTO, T.; LEE, K. G. Identification of volatile components in basil (Ocimum basilicum L.) and thyme leaves (Thymus vulgaris L.) and their antioxidant properties. Food Chemistry, London, v. 91, n. 1, p. 131-137, 2005. http:// dx.doi.org/10.1016/j.foodchem.2004.05.056.
MARTINELLO, M. A.; MUÑOZ, D. J.; GINER, S. A. Mathematical modelling of low temperature drying of maize: comparison of numerical methods for solving the differential equations. Biosystems Engineering, Harpenden, v. 114, n. 2, p. 187-194, 2013. http://dx.doi.org/10.1016/j.biosystemseng.2012.11.014.

MENSOR, L. L.; MENEZES, F. S.; LEITAO, G. G.; REIS, A. S.; SANTOS, T. C.; COUBE, C. S.; LEITÃO, S. G. Screening of Brazilian plant extracts for antioxidant activity by the use of DPPH free radical method. Phytoterapy Research, London, v. 15, n. 2, p. 127-130, 2001. http://dx.doi.org/10.1002/ptr.687. PMid:11268111.

MERCADANTE, A. Z.; RODRIGUEZ-AMAYA, D. B.; BRITTON, G. HPLC and mass spectrometric analysis of carotenoids from mango. Journal of Agriculture Food and Chemistry, London, v. 45, n. 1, p. 120-123, 1997. http://dx.doi.org/10.1021/jf960276j.

OLIVEIRA, E. G.; ROSA, G. S.; MORAES, M. A.; PINTO, L. A. A. Characterization of thin layer drying of Spirulina platensis utilizing perpendicular air flow. Biosource Technology, Kidlington, v. 100, n. 3, p. 1297-1303, 2009. http://dx.doi.org/10.1016/j. biortech.2008.05.052. PMid:18922691.

OREN-SHAMIR, M. Does anthocyanin degradation play a significant role in determining pigment concentration in plants? Plant Science, Davis, v. 177, n. 4, p. 310-316, 2009. http:// dx.doi.org/10.1016/j.plantsci.2009.06.015.

RASEIRA, M. C. B. A pesquisa com amora-preta no Brasil. In: SIMPÓSIO NACIONAL DO MORANGO, 2.; ENCONTRO DE PEQUENAS FRUTAS E FRUTAS NATIVAS, 2004, Pelotas. Palestra.. Pelotas: Embrapa Clima Temperado, 2004. p. 219-223.

ReÁtegui, J. L. P.; MAChAdO, A. P. F.; BARBERO, G. G.; REZENDE, C. A.; MARTíNEZ, J. Extraction of antioxidant compounds from blackberry (Rubus SP) bagasse using supercritical $\mathrm{CO} 2$ assisted by ultrasound. The Journal of Supercritical Fluids, Blacksburg, v. 94, p. 223-233, 2014. http://dx.doi.org/10.1016/j. supflu.2014.07.019.

ROMERO JUNIOR, C. A.; VILLAREAL, B. D. Y. Ultrasound as pretreatment to convective dryng of andeand blackberry (Rubus glaucus Benth). Ultrasonic Sonochemistry, Coventry, v. 22, p. 2015-2210, 2015.

SGARBIERI, V. C. Nutrição e tecnologia de alimentos. Boletim da Sociedade Brasileira de Ciência e Tecnologia de Alimentos, Campinas, v. 20, p. 115-139, 1986.

SILVA, J. S. Secagem e armazenamento de produtos agrícolas. Viçosa: Aprenda Fácil, 2003. p. 39-62.

STRIK, B. C.; CLARK, J. R.; FINN, C. E.; BAÑADOS, M. P. Worldwide blackberry production. Hortechnology, Alexandria, v. 17, n. 2, p. 205-213, 2007.

TULLIO, L.; AYUB, R. A. Produção da amora-preta cv tupy, em função da intensidade da poda. Semina: Ciências Agrárias, Londrina, v. 34, n. 3, p. 1147-1152, 2013. 
Planejamento experimental do processo de secagem da amora-preta (Rubus sp.) para a produção de farinha enriquecida com compostos bioativos

Casarin, F. et al.

WANG, S. Y.; BOWMAN, L.; DING, M. Methyl jasmonate enhances antioxidant activity and flavonoid content in blackberries (Rubus spp) and promotes antiproliferations of human cancer cells. Food Chemistry, London, v. 107, n. 3, p. 1261-1269, 2008.
ZHISHEN, J.; MENGCHENG, T.; JIANMING, W. The determination of flavonoid contents in mulberry and their scavenging effects on superoxide radical. Food Chemistry, London, v. 64, n. 4, p. 555-559, 1999. http://dx.doi.org/10.1016/S0308-8146(98)00102-2. 